\title{
Vorwort und Benutzungshinweise zur 12. Auflage
}

Aus der Betreuung und Begutachtung ungezählter Seminararbeiten und von inzwischen weit über 1.000 Diplom- sowie Bachelor- und Masterarbeiten ist unser Eindruck: Studierende fühlen sich bei der Anfertigung wissenschaftlicher Arbeiten hinsichtlich der Frage, was wissenschaftliches Arbeiten eigentlich bedeutet und ausmacht, zunächst sehr unsicher. Vielen mangelt es von Grund auf an Kenntnissen, die die Voraussetzung für erfolgreiches wissenschaftliches Arbeiten darstellen: Die schulische Ausbildung hat ihnen dazu nichts vermittelt. Und nicht in allen Studiengängen werden sie hierauf gezielt vorbereitet, bevor sie ihre erste wissenschaftliche Arbeit schreiben. Manchmal werden zwar Techniken und Regeln wissenschaftlichen Arbeitens vermittelt, aber ohne deren tieferen Sinn zu erklären. Dies führt dazu, dass Studierende ratlos vor dem Wust an Zitierregeln oder Regeln zur Auswahl angemessener Quellen stehen, Angst haben, aus Unwissenheit Plagiate zu begehen, oder nicht einschätzen können, warum bzw. wann sie welche Regel anwenden sollten oder wie diese in Zweifelsfällen auszulegen ist.

Mit diesem Buch bieten wir in konzentrierter Form umfassende Hilfestellung auf verschiedenen Ebenen:

- Teil A „Grundansprüche an wissenschaftliche Arbeiten“ vermittelt, was wissenschaftliches Arbeiten ausmacht und welche grundlegenden Anforderungen Arbeiten zu erfüllen haben, um als ,wissenschaftlich' gelten zu können. Über diese generellen Anforderungen sollte Klarheit herrschen, denn sie bilden das Fundament wissenschaftlichen Arbeitens und sind die Grundlage der Regeln und Techniken wissenschaftlichen Arbeitens. Es bietet sich an, diesen Teil des Buches zu lesen, bevor man mit der Arbeit an einem wissenschaftlichen Text beginnt.

- Teil B „Entstehungsprozess einer wissenschaftlichen Arbeit“ behandelt Hinweise und Regeln für die einzelnen Entstehungsphasen einer wissenschaftlichen Arbeit und konkretisiert die Technik des wissenschaftlichen Arbeitens über Demonstrationsbeispiele. Teil B kann gut begleitend zu der Arbeit am wissenschaftlichen Text gelesen werden und als Nachschlagewerk zu Detailfragen und -problemen während der konkreten Recherche- und Schreibarbeit verwendet werden.

- Teil C „Beurteilungskriterien für wissenschaftliche Arbeiten und Regeln guter wissenschaftlicher Praxis“ fasst die Grundansprüche und Regeln zusammen, indem Kriterien präsentiert werden, an denen Gutachterinnen und Gutachter ihre Bewertung häufig ausrichten. Teil C fasst solche Kriterien und Regeln in einer Art ,Gesamt-Checkliste‘ zusammen. An ihr lässt sich prüfen, ob die zur 
Abgabe vorgesehene Fassung wirklich in jeder Hinsicht der Technik und den Regeln wissenschaftlichen Arbeitens entspricht und alle Grundansprüche erfüllt. Daher bietet es sich an, Teil C kurz vor Abgabe der Arbeit zur Überprüfung des fast fertigen Werkes zu nutzen.

Für diese 12. Auflage wurde die gesamte Schrift gründlich durchgesehen und durchlaufend sprachlich und inhaltlich aktualisiert. Formulierungen wurden präzisiert und Druckfehler korrigiert. Ein Abschnitt zur Grundstruktur unterschiedlicher Typen von wissenschaftlichen Arbeiten wurde neu aufgenommen. Die Ausführungen zum Sprachgebrauch bezüglich der Ich- und Wir-Perspektive der Autoren wurden aktualisiert. Die Abschnitte zur Zitierweise wurden stärker als in früheren Auflagen auf die heute aktuelle textintegrierte Kurzbeleg-Zitierweise ausgerichtet. Deutlich erweitert und unter Co-Autorschaft von Herrn M. Sc. Tobias Moll neu geschrieben wurden auch die Abschnitte zur Literaturrecherche und -selektion.

Wir bedanken uns sehr herzlich bei Aylin Satmaz, Lynn Koltermann und Annika Scheele, die uns unterstützt haben beim Korrekturlesen dieser neuen Auflage - und mit ihren Verbesserungsvorschlägen und Anmerkungen zur weiteren Verbesserung des Buches beigetragen haben.

Wir wünschen Ihnen viel Freude beim ,Wissenschaftlichen Arbeiten ${ }^{\star}$ - und viel Erfolg mit Ihren wissenschaftlichen Arbeiten! 